\title{
Vaginal discharge in prepuberal girls
}

Volume 4 Issue 5 - 2016

\author{
Adil Elghanmi, Amina Lakhdar \\ Faculte de Medecine et de Pharmacie Universite Souissi, \\ Morocco
}

\section{Letter to editor}

Dear Editor, vaginal discharge in prepuberal girls is rare and may have many different etiologies. A 7 years old girl was brought to the Emergency Department of Gynecology and Obstetrics Rabat, Morocco by her mother, for recurrent vaginal discharge. This young girl had no medical or surgical history. Physical examination revealed a healthy, afebrile patient. Genital examination in the frog-leg position revealed a vulvar erythema with malodorous discharge. They were no tears or abnormalities on the hymen and both labia. On rectal examination a small round and solid mass was palpated.

An antero-posterior radiography of the pelvis showed two radio opaque foreign bodies projecting over the pelvic inlet (Figure 1). The girl was cooperative and the foreign bodies were removed by a digital rectal maneuver that consisted of expulsing the objects from the vagina by applying anantero-posterior pressure on the vaginal posterior wall. The foreign bodies were battery watch (Figure 2).

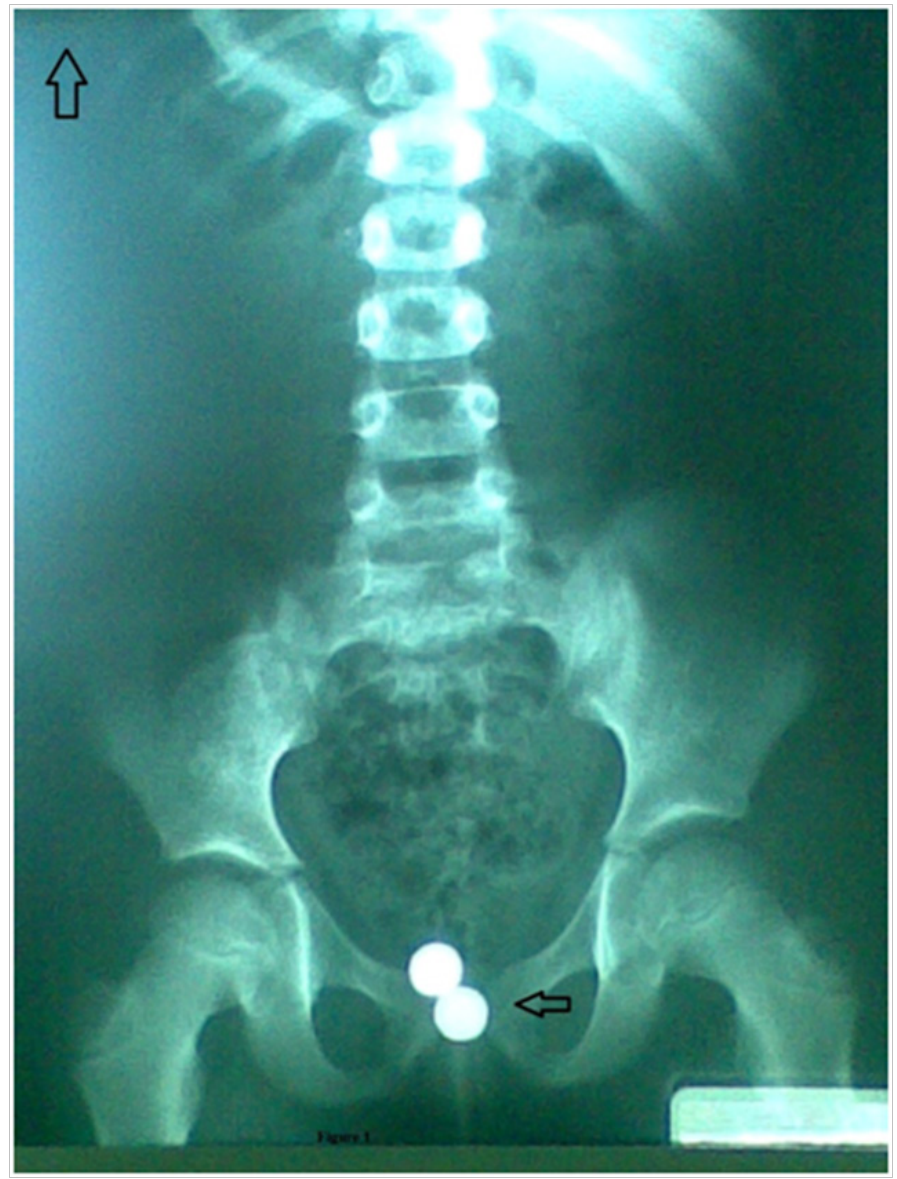

Figure I Antero-posterior radiography.

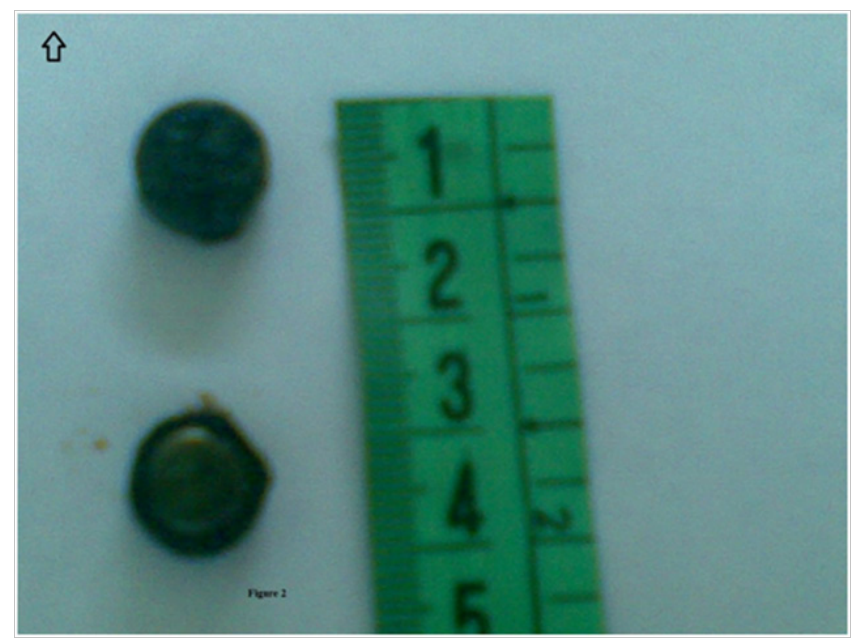

Figure 2 Foreign bodies.

The child was treated with metronidazole. The follow up was uneventful and the girl was seen on the outpatient department at two and four weeks with no clinical complication. The diagnosis of prepuberal vaginal discharges includes nonspecific vulvovaginitis, precocious puberty, anatomical anomaly and sexual abuse. Although, vaginal foreign bodies should be evocated in the presence of recurrent or permanent vaginal discharge in prepuberal patients. The diagnostic procedures include non invasive imaging as plain radiography, ultrasonography and MRI. In case of negative findings vaginal irrigation and/or diagnostic vaginoscopy should be considered. ${ }^{1}$ The foreign body can be removed easily like in our case, or by the use of a narrow illuminated endoscope. ${ }^{2}$ Even though rare, the possibility of foreign body in the vagina should be kept in mind while examining any child with vulvovaginal symptoms. 


\section{Acknowledgements}

None.

\section{Conflicts of interest}

None.

\section{References}

1. Gobbur VR, Gobbur RH, Patil AG, Endigeri P. A rare case of foreign body causing recurrent vaginal discharge in prepubertal child. J Clin Diagn Res. 2015;9(1):QD03-QD04.

2. Sakhavar N, Teimoori B, Ghasemi M. Foreign body in the vagina of a four-year-old-girl: a childish prank or sexual abuse. Int J High Risk Behav Addict 21. 2014;3(2):e10534. 